Fitt, A

Generalized exact solutions for boundary layer flow and heat transfer over permeable stretching sheets

Fitt, A (2012) Generalized exact solutions for boundary layer flow and heat transfer over permeable stretching sheets. Applied Mathematics and Computation, 219 (4). pp. 1468-1473.

doi: 10.1016/j.amc.2012.07.049

This version is available: https://radar.brookes.ac.uk/radar/items/0b7d9387-e136-425a-abcd-28b144ff9c59/1/

Available on RADAR: November 2016

Copyright (C) and Moral Rights are retained by the author(s) and/ or other copyright owners. A copy can be downloaded for personal non-commercial research or study, without prior permission or charge. This item cannot be reproduced or quoted extensively from without first obtaining permission in writing from the copyright holder(s). The content must not be changed in any way or sold commercially in any format or medium without the formal permission of the copyright holders.

This document is the post print version of the journal article. Some differences between the published version and this version may remain and you are advised to consult the published version if you wish to cite from it. 


\title{
Generalized exact solutions for boundary layer flow and heat transfer over permeable stretching sheets
}

\author{
A.D. Fitt \\ Oxford Brookes University, Headington Campus, Gipsy Lane, Oxford OX3 OBP, UK
}

\begin{abstract}
The flow of a viscoelastic fluid over a porous stretching sheet is considered in the presence of a transverse magnetic field, volumetric heating, heat transfer, and a range of other effects. By observing the essential mathematical mechanism by which exact solutions have been generated in many previous studies, it is shown how a framework may be developed that both unifies and generalizes previously-reported closed-form solutions to such problems. It is further shown how, by judicious choices of the arbitrary functions involved, novel exact solutions that do not seem to have appeared previously may be generated.
\end{abstract}

\section{Introduction}

Many recent papers have dealt with the two-dimensional boundary layer flow of a viscous incompressible fluid over a moving (sometimes described as "stretching") flat plate, observing that under certain special circumstances it is possible to give closed-form solutions to the equations of motion. Such exact solutions normally require that the wall is porous, and that the vertical component of velocity behaves in a given (non-zero) manner at the wall. Though it may be argued that such flows are not often encountered in physically relevant problems, there is no doubt that in some industrial processes that involve (for example) extrusion, hot rolling, the drawing of wires and sheets of fabric (for example, in glass fibre production) and various types of heat treatment, analysis of such flows (and the heat transfer associated with them) may be valuable. Further, the flow fields that are predicted under such circumstances may serve as useful tests of boundary layer codes, and can give insights into the general nature of boundary layer flows. 
Though a great many previous studies have considered flows of this type, this general area of investigation appears to have been begun by the studies [1], [2] and [3]. A wide range of subsequent papers has covered many different aspects of such flows, introducing a plethora of extra terms and effects. Many of these previous studies have alluded to various exact solutions that exist.

The purpose of this short note is to show (i) the mathematical reasons why the exact solutions that have been derived in the past have been possible, (ii) how a great deal of the various different cases that have previously been dealt with can be considered using a common framework, rather than as a sequence of incremental special cases (iii) in what circumstances other effects may be included, and what functional forms the extra effects must take to enable an exact solution to exist and (iv) how, using the framework that is developed, a range of new and apparently previously unreported exact solutions may be derived.

\section{Governing equations}

We assume that steady incompressible boundary layer flow is taking place in $y>0$ with velocity $\mathbf{q}=(u, v)$ and a pressure gradient $p_{x}$. The general equations of motion for the flow and heat transfer may be written in the form

$$
\begin{gathered}
\rho\left(\boldsymbol{q}_{t}+(\boldsymbol{q} \cdot \nabla) \boldsymbol{q}\right)=-\nabla p+\nabla \cdot S+\rho \boldsymbol{f} \\
\nabla \cdot \boldsymbol{q}=0 \\
\rho c_{p}\left(T_{t}+(\boldsymbol{q} \cdot \nabla) T\right)=k \nabla^{2} T+\Phi .
\end{gathered}
$$

Here $\rho$ denotes density, $t$ denotes time, $S$ is the viscous part of the stress tensor $\tau_{i j}\left(\tau_{i j}=S_{i j}-p \delta_{i j}\right)$, $\rho \boldsymbol{f}$ is the body force per unit volume, $T(x, y)$ denotes temperature, $c_{p}$ denotes specific heat at constant pressure, $k$ denotes thermal conductivity and $\Phi$ denotes any extra thermal effects that might arise from viscous dissipation, buoyancy or body heating.

The equations (1)-(3) must now be written in a suitable dimensionless form and the usual boundary layer arguments must be applied (we omit the details for brevity). We also introduce that a stream function $\psi(x, y)$ defined in the normal manner. The governing equations may then be written as

$$
\begin{gathered}
\psi_{y} \psi_{x y}-\psi_{x} \psi_{y y}+p_{x}-K_{\eta} \psi_{y y y}=-K_{m} \psi_{y}+ \\
K_{\tau}\left[\psi_{y} \psi_{x y y y}-\psi_{x} \psi_{y y y y}+\psi_{x y} \psi_{y y y}-\psi_{y y} \psi_{x y y}\right]
\end{gathered}
$$




$$
\psi_{y} T_{x}-\psi_{x} T_{y}-K_{h} T_{y y}=-K_{v}\left(T-T_{\infty}\right)+K_{d} \psi_{y y}^{2}-K_{c} T^{n}
$$

Where $T_{\infty}$ is a suitably non-dimensionalized reference temperature. The exact number of boundary conditions required for these equations depends on whether or not $K_{\tau}$ is zero, but in all cases the no-slip condition is applied at the plate $y=0$, we insist that $\psi_{y} \rightarrow 0$ as $y \rightarrow \infty$, and either the heat flux or the surface temperature is specified at $y=0$.

Various authors in various previous studies have included, excluded or modified terms in (4) and (5) corresponding to various choices of models for $S_{i j}, \boldsymbol{f}$ and $\Phi$. Previous modelling assumptions have included setting $p_{x}=0$ to study zero pressure gradient boundary layers (the reason for this will soon become apparent) setting $K_{m} \neq 0$ to simulate (via the simplest MHD modelling assumptions available) the MHD effects of a uniform magnetic field along the $y$-axis or to simulate the flow through a highly permeable or saturated porous medium (see, for example, [4] or [5] respectively), setting $K_{\tau} \neq 0$ to include the effects of viscoelasticity via various classical models (see, for example, [6], which adopts a standard model of viscoelasticity), setting $K_{v} \neq 0$ to simulate the effects of volumetric heat absorption by assuming that the heat gained is proportional to the temperature difference (see, for example [7]), setting $K_{c} \neq 0$ to simulate the effects of chemical reaction (when the temperature $T$ in (5) is replaced by a concentration $c(x, y)$ - see, for example, [8]), setting $K_{d} \neq 0$ to include the effects of viscous dissipation in the boundary layer and modifying the heat diffusivity term $K_{h}$ to include the effects of bulk radiative heat transfer to an optically thick material using the Rosseland approximation model (see, for example, [9]).

Other terms too numerous to mention have also been added to simulate extra flow effects (for example, a simple Boussinesq buoyancy model adds a term proportional to $T-T_{\infty}$ to (4)), and in some studies (see, for example, [10]) a concentration equation and an energy equation are solved simultaneously. Different kinds of non-Newtonian behaviour (e.g. pseudoplasticity) have also previously been considered. In other studies (see, for example, [11]) extra equations for extra quantities (for example, the micro-rotation in a micropolar fluid) have been added: in most cases however the extra terms enter the equations in a linear manner and so could be included in the anaysis presented below if required.

\subsection{Simplification of nonlinear terms - momentum equation}

The equations (4) and (5) are nonlinear, and almost all previous relevant studies that have presented exact solutions have relied on the fact that these 
solutions are of such a form that the nonlinear terms either exactly cancel, or produce a result that is linear in the unknown functions and takes a particularly simple and convenient form. To investigate this, we first note that (4) may be rewritten as

$$
M-K_{\tau}\left(M_{y y}-2 N\right)+p_{x}-K_{\eta} \psi_{y y y}=-K_{m} \psi_{y}
$$

where

$$
M=\psi_{y} \psi_{x y}-\psi_{x} \psi_{y y}=\psi_{y}^{2}\left(\frac{\psi_{x}}{\psi_{y}}\right)_{y}, \quad N=\psi_{y y}^{2}\left(\frac{\psi_{x y}}{\psi_{y y}}\right)_{y}
$$

so that the "linearisation" of the equation amounts to the fact that

$$
M-K_{\tau}\left(M_{y y}-2 N\right)=C(\psi)
$$

where $C(\psi)$ is a linear function of $\psi$ and/or its partial derivatives. Though no doubt there are many ways in which (6) may hold, many of the previouslystudied exact solutions rely to a great extent on results closely related to the case when $M=0$. If the stream function $\psi$ is to be chosen so that $M=0$, then either $\psi_{y}=0$ (in which case there is no flow) or $\psi_{x} / \psi_{y}$ must be a function of $x$ alone. The latter may be viewed as a quasilinear first order partial differential equation for $\psi$, having general solution $\psi(x, y)=F(y-f(x))$ where both $F$ and $f$ are arbitrary functions of a single variable. It transpires that for most useful exact solutions having $M=0$ is slightly too restrictive, and small modifications give more interesting solutions. We therefore note that (6) is rendered linear (but non zero) if an arbitrary function $q(x)$ is added to $F(y-f(x))$. In this case, with

$$
\psi(x, y)=\psi_{0}(x, y)=q(x)+F(y-f(x))
$$

we have

$$
M=-q^{\prime}(x) F^{\prime \prime}(y-f(x)), \quad N=0
$$

so that

$$
M-K_{\tau}\left(M_{y y}-2 N\right)=-q^{\prime}(x) F^{\prime \prime}(y-f(x))+K_{\tau} q^{\prime}(x) F^{\prime \prime \prime \prime}(y-f(x))
$$

and

$$
u(x, 0)=u_{w}(x)=F^{\prime}(-f(x)), \quad v(x, 0)=v_{w}(x)=-q^{\prime}(x)+f^{\prime}(x) F^{\prime}(-f(x)) .
$$


In general therefore such exact solutions apply to a flat plate that is moving with horizontal speed $F^{\prime}(-f(x))$ and is porous, so that there is either sucking or blowing at the wall. When the choice $\psi=\psi_{0}$ is used, the momentum equation reduces to

$$
-q^{\prime}(x) F^{\prime \prime}(s)+p^{\prime}(x)-K_{\eta} F^{\prime \prime \prime}(s)+K_{m} F^{\prime}(s)+K_{\tau} q^{\prime}(x) F^{\prime \prime \prime \prime}(s)=0
$$

(where $s=y-f(x)$ ) which is linear in $F(s)$ : as a result, further progress may now be made.

\subsection{Vanishing of nonlinear terms - energy equation}

We now turn to the energy equation (5). Using $\psi=\psi_{0}$ from (7) this becomes

$F^{\prime}(s) T_{x}+\left(F^{\prime}(s) f^{\prime}(x)-q^{\prime}(x)\right) T_{y}=K_{h} T_{y y}-K_{v}\left(T-T_{\infty}\right)+K_{d}\left(F^{\prime \prime}(s)\right)^{2}+K_{c} T^{n}$.

This is linear in $T(x, y)$ (apart from the possible inclusion of the chemical reaction term $c_{n} T^{n}$ ) but in general requires the solution of an advectiondiffusion equation. Closed-form solutions are therefore hard to derive. so many previous studies have now made progress by treating this equation in a similar fashion to the momentum equation, and seeking solutions in which the terms $\psi_{y} T_{x}-\psi_{x} T_{y}$ either identically vanish or become a great deal simpler. This happens most easily when we note that

$$
\psi_{y} T_{x}-\psi_{x} T_{y}=\psi_{x} T_{x}\left(\frac{\psi_{y}}{\psi_{x}}-\frac{T_{y}}{T_{x}}\right),
$$

an expression that vanishes when we use (7) and solve (9) for $T$ to yield $T(x, y)=G(F(s)+q(x))$ where $G$ is another arbitrary function. Again, we find that the most interesting exact solutions tend to occur when (9) is not quite zero, but has a slightly more general form. For example, the energy equation remains linear if an arbitrary function $b(x)$ is added to this solution. Writing $F(s)+q(x)=t$ for simplicity, we note that if

$$
T(x, y)=T_{0}(x, y)=G(F(s)+q(x))+b(x)=G(t)+b(x)
$$

then

$$
u T_{0 x}+v T_{0 y}=b^{\prime}(x) F^{\prime}(s) .
$$


The governing equation also remains linear if, for arbitrary functions $H(s)$ and $c(x)$, we take

$$
T(x, y)=T_{1}(x, y)=H(s)+c(x)
$$

since then

$$
u T_{1 x}+v T_{1 y}=c^{\prime}(x) F^{\prime}(s)-q^{\prime}(x) H^{\prime}(s) .
$$

\section{Framework for previous studies}

Thus far, we have expounded and clarified the general method that has previously been used in a large number of special cases to derive exact solutions to a wide range of problems involving boundary layers over a permeable stretching sheet. We now examine various choices for the arbitrary functions $F, G, H, q, b, c$ and $f$ that are at our disposal.

\subsection{Flows where $F$ is an exponential}

When there is no pressure gradient so that $p(x)=0$, the simplest choice for the arbitrary function $F$ is an exponential. Though this choice has often been made before, many previous studies chose to treat particular special cases of $f(x)$ (such as linearly, quadratically or exponentially stretching sheets) rather than to explore the general case. Choosing $F(s)=-e^{-k s}$, and $f(x)=(1 / k) \log \left(u_{w}(x) / k\right)$ in $(7)$ we find that, for arbitrary $u_{w}(x)$,

$$
\psi(x, y)=q(x)-\frac{u_{w}(x)}{k} e^{-k y}
$$

where, from $(8), q(x)$ must satisfy

$$
q^{\prime}(x)\left(-K_{\tau} k^{3}+k\right)-K_{\eta} k^{2}+K_{m}=0 .
$$

Thus (surpressing the arbitrary constant in $\psi(x, y)$ )

$$
\begin{gathered}
\psi(x, y)=Q x-\frac{u_{w}(x)}{k} e^{-k y}, \quad u(x, y)=u_{w}(x) e^{-k y}, \\
v(x, y)=-Q+\frac{u_{w}^{\prime}(x)}{k} e^{-k y}
\end{gathered}
$$

where

$$
Q=\frac{K_{\eta} k^{2}-K_{m}}{k\left(1-K_{\tau} k^{2}\right)}
$$


and $u \rightarrow 0$ as $y \rightarrow \infty$ for $\operatorname{Re}(k)>0$. The plate $y=0$ is impermeable only in the case when $u_{w}(x)=k Q x+$ constant . This solution seems to have been reported first (in the special case $K_{\tau}=K_{m}=0, p_{x}=0$ ) in [12], and provides a generalization of previous studies such as [2], [3], [13], [14], [15], and a great many others that deal with a huge variety of special cases.

Let us now turn to the solution to the energy equation when $F$ is an exponential. With $F(s)=-e^{-k s}$ and $f(x)=(1 / k) \log \left(u_{w}(x) / k\right)$, we find that picking $H(s)=C \exp (\mu(\log k-s k))$ (where $C$ is an arbitrary constant) and $c(x)=T_{\infty}$ in (10), the energy equation (5) with $K_{d}=K_{c}=0$ reduces to

$$
K_{h} \mu^{2} k^{2}-Q k \mu-K_{\nu}=0 .
$$

Thus an exact solution is generated if

$$
\mu=\frac{Q \pm \sqrt{Q^{2}+4 K_{h} K_{\nu}}}{2 k K_{h}}
$$

in which case

$$
T(x, y)=T_{\infty}+C u_{w}(x)^{\mu} e^{-\mu k y}
$$

This solution provides a generalization of the heat transfer solution first reported in [12] and does not appear to have been previously discussed in the literature. Since $k>0$ so that $u \rightarrow 0$ as $y \rightarrow \infty$, the solution is only valid if $\mu>0$ so that $T \rightarrow T_{\infty}$ as $y \rightarrow \infty$. We note that $K_{h}>0$ (or (4) is unstable), so if $K_{\nu}<0$ then there may be circumstances under which the solution (11) ceases to be valid, either because $\mu$ is not real, or (when $Q<0$ ) there are no positive values of $\mu$.

\subsection{Flows where $F$ is not an exponential}

A range of exact solutions is also possible when the function $F$ is chosen to take a more complicated form than a simple exponential. Many examples may be examined, but a simple illustration of such a flow is given by the choice $F(s)=-k s e^{-k s}, q(x)=A x$ where $A$ is constant. Using this in (7) with arbitrary $f(x)$, the momentum equation (4) with no pressure gradient becomes

$k(-y+f(x))\left(K_{\tau} A k^{3}+K_{\eta} k^{2}-A k-K_{m}\right)+4 K_{\tau} A k^{3}+3 K_{\eta} k^{2}-2 A k-K_{m}$.

Setting $A=Q$, we find that an exact solution is generated so long as $k$ satisfies

$$
-K_{\tau} K_{\eta} k^{4}+3 K_{\tau} K_{m} k^{2}-k^{2} K_{\eta}-K_{m}=0
$$


This quadratic equation for $k^{2}$ yields a variety of roots for $k$ that may take different real and imaginary values depending on the signs and values of the various parameters involved. In many cases however interesting and practical exact solutions exist. For example, in the special case $K_{\eta}=1, K_{m}=10$, $K_{\tau}=1 / 9$, we find that a solution of (4) is given, for arbitrary $f(x)$, by

$$
\psi(x, y)=\frac{9\left(10-k^{2}\right) x}{k\left(k^{2}-9\right)}+k(-y+f(x)) e^{-k(y-f(x))}
$$

where $k$ can take either of the values $\sqrt{6}$ or $\sqrt{15}$. For either of these values of $k$ the velocity components are given by

$$
\begin{gathered}
u=k(k(y-f(x))-1) e^{-k(y-f(x))}, \\
v=\frac{9\left(k^{2}-10\right)}{k\left(k^{2}-9\right)}+k f^{\prime}(x)(k(y-f(x))-1) e^{-k(y-f(x))},
\end{gathered}
$$

so that the $y$-dependence is not simply exponential, and these solutions are of an essentially different form to those derived in Section 3.1.

It is also possible to generate solutions of the energy equation (5) for this choice of functions. For example, when $K_{\eta}=1, K_{\tau}=5 / 3$ and $K_{m}=2 / 3$ so that $Q=-1 / 2$ and $k=1$, the stream function is given, for arbitrary $f(x)$, by

$$
\psi(x, y)=-\frac{x}{2}+(y-f(x)) e^{-(y-f(x))}
$$

and, it may easily be confirmed that an exact solution in the case $K_{d}=K_{c}=$ 0 is given by

$$
T(x, y)=T_{\infty}+G(y-f(x))
$$

where

$$
G(s) \propto \exp \left(\frac{-s\left(-1+\sqrt{1+16 K_{\nu} K_{h}}\right)}{4 K_{h}}\right) .
$$

Many other new exact solutions may be generated using other choices of $F(s)$ and the functions at our disposal: we do not pursue these further, however, for our purpose here has not been to try to give large numbers of examples, but rather to suggest a methodology for generating such solutions. 


\subsection{Unsteady flows}

It has already been observed that stream functions of the form (7) essentially linearise (4). For this reason, it is possible to use the methodology developed above to generate closed-form solutions to the unsteady versions of (4) and (5), namely

$$
\begin{gathered}
\psi_{y t}+\psi_{y} \psi_{x y}-\psi_{x} \psi_{y y}+p_{x}-K_{\eta} \psi_{y y y}=-K_{m} \psi_{y}+ \\
K_{\tau}\left[\psi_{y} \psi_{x y y y}-\psi_{x} \psi_{y y y y}+\psi_{x y} \psi_{y y y}-\psi_{y y} \psi_{x y y}\right] \\
T_{t}+\psi_{y} T_{x}-\psi_{x} T_{y}-K_{h} T_{y y}=-K_{v}\left(T-T_{\infty}\right)+K_{d} \psi_{y y}^{2}-K_{c} T^{n}
\end{gathered}
$$

Under normal circumstances the essential nonlinearity of the boundary-layer equations means that it is particularly challenging to derive closed-form solutions to the unsteady equations, but because of the observations in Sect. 2.1, stream functions of the form

$$
\psi(x, y, t)=\psi_{u}(x, y, t)=q(x)+F(y-f(x)) r(t)
$$

transform (12) (with $p(x)=0)$ to

$$
r^{\prime}(t) F^{\prime}(s)+r(t)\left(-q^{\prime}(x) F^{\prime \prime}(s)-K_{\eta} F^{\prime \prime \prime}(s)+K_{m} F^{\prime}(s)+K_{\tau} q^{\prime}(x) F^{\prime \prime \prime \prime}(s)\right)=0
$$

and closed-form solutions may now easily be generated. For example, choosing $r(t)=e^{-\zeta t}$, and once again using the choices $F(s)=-e^{-k s}$ and $f(x)=$ $(1 / k) \log \left(u_{w}(x) / k\right)$, we find that an unsteady solution of the form

$$
\psi(x, y, t)=Q x-\frac{1}{k} u_{w}(x) e^{-k y-\zeta t}
$$

exists if

$$
Q=\frac{-\zeta-K_{\eta} k^{2}+K_{m}}{k\left(-1+K_{\tau} k^{2}\right)}
$$

A range of related exact solutions to the unsteady energy equation (13) may now be generated in an obvious fashion.

Again, though there are many relatively simple ways in which (14) may be generalised, we have not sought to include every type of possible unsteady closed-form solution in the discussion above, but have rather preferred to simply indicate the general type of methodology that may be used to generate closed-form solutions of various types. Note also that the relative rarity of unsteady exact boundary layer solutions means that solutions like (16) may prove extremely useful as test cases for code development and verification. 


\subsection{Flows with a pressure gradient}

No previous studies of exact solutions for boundary layer flow over stretching sheets appear to have considered cases where the pressure gradient is nonzero. Let us recall that with $\psi(x, y)$ given by $(7)$, the momentum equation becomes

$$
-q^{\prime}(x) F^{\prime \prime}(s)+p^{\prime}(x)-K_{\eta} F^{\prime \prime \prime}(s)+K_{m} F^{\prime}(s)+K_{\tau} q^{\prime}(x) F^{\prime \prime \prime \prime}(s)=0 .
$$

The only instance where it is possible to choose functions $F, f$ and $q$ so that the quantity

$$
\xi=-q^{\prime}(x) F^{\prime \prime}(s)-K_{\eta} F^{\prime \prime \prime}(s)+K_{m} F^{\prime}(s)+K_{\tau} q^{\prime}(x) F^{\prime \prime \prime \prime}(s)
$$

is independent of $y$ appears to occur when $F(s)$ is proportional to $s$. Since this does not lead to a flow where $u \rightarrow 0$ as $y \rightarrow \infty$ such examples must be discounted. Thus no exact solutions of this form with non-zero pressure gradient seem possible. Exact solutions of a different form may exist, but they cannot arise from solutions of the form (7) and require a more complicated linearisation of (6). One example of such solutions is given by Falkner-Skan type flows where the pressure is proportional to $x^{k}$ and the stream function is of the form

$$
\psi(x, y)=x^{m} F\left(y x^{n}\right)
$$

where $m=1+n, k=2+4 n$. Here though a closed-form solution seems to be available only if $n=-1$ and $K_{m}=K_{\tau}=0$.

\section{Summary and conclusions}

A general framework has been given that not only encompasses, unifies and generalizes a wide range of exact solutions that have appeared in a very large number of previous publications, but also leads to a variety of new exact solutions that do not seem to have been reported before. The framework identifies the key reason why exact solutions are possible in these cases, and shows how a range of choices may be made that lead to different and novel exact solutions.

Using this framework, a large variety of exact solutions may be generated to use as test cases, paradigm problems for various flows involving stretching porous plates in MHD and heat transfer-influenced non-Newtonian flows, and these may easily be extended to cases where other effects (such as buoyancy) 
are included. Though space does not allow a full consideration of all of these effects, there is little doubt that a wide range of physically-relevant cases may be examined using this methodology.

Many other closed-form solutions may be investigated. To give one final example, it is easily verified that useful simplifications of (6) also take place if $\psi(x, y)=F(y-f(x))+\zeta(y)$ and the choices $F(s)=e^{-k s}$ and $f(x)=\log (x)$ are made. In this case generalisations of the exact solution reported in [16] may be generated. Flows with a non-zero pressure gradient appear to be harder to treat, and though it does not appear that any simple exact solutions are currently available, there is no reason why other simplifications of (6) might not eventually yield novel exact solutions for flows with a pressure gradient and many other kinds of more complicated flow.

\section{References}

[1] B.C. Sakiadis, Boundary-layer behaviour on continuous solid surfaces. AIChE J. 7 (1961) 26-28.

[2] L.J. Crane, Flow past a stretching plate. Z. Angew. Math. Phys. 21 (1970) 645-647.

[3] P.S. Gupta, A.S. Gupta, Heat and mass transfer on a stretching sheet with suction or blowing. Can. J. Chem. Eng. 55 (1977) 744-746.

[4] H.I. Andersson, MHD flow of a viscoelastic fluid past a stretching surface. Acta Mech. 95 (1992) 227-230.

[5] K. Vajravelu, Flow and heat-transfer in a saturated porous-medium over a stretching surface. Z. Angew. Math. Mech. 74 (1994) 605-614.

[6] P.D. Areil, MHD flow of a viscoelastic fluid past a stretching sheet with suction. Acta Mech. 105 (1994) 49-56.

[7] P.S. Lawrence, B.N. Rao, Heat transfer in a viscoelastic boundary layer flow over a stretching sheet. J. Phys. D Appl. Phys. 27 (1994) 1323-1327.

[8] R. Cortell, MHD flow and mass transfer of an electrically conducting fluid of second grade in a porous medium over a stretching sheet with chemically reactive species. Chem. Eng. Process. 46 (2007) 721-728. 
[9] R. Cortell, Heat and fluid flow due to non-linearly stretching surfaces. Appl. Math. Comput. 217 (2011) 7564-7572.

[10] N. Bachok, A. Ishak, I. Pop, Boundary-layer flow of nanofluids over a moving surface in a flowing fluid. Int. J. Therm. Sci. 49 (2010) 16631668 .

[11] E. Magyari, A.J. Chamkha, Combined effect of heat generation or absorption and first-order chemical reaction on micropolar fluid flows over a uniformly stretched permeable surface: The full analytical solution. Int. J. Therm. Sci. 49 (2010) 1821-1828.

[12] P. Weidman, E. Magyari, Generalized Crane flow induced by continuous surfaces stretching with arbitrary velocities. Acta Mech. 209 (2010) 353362 .

[13] N. Ahmad, Visco-elastic boundary layer flow past a stretching plate and heat transfer with variable thermal conductivity. W. J. Mech. 1 (2011) $15-20$.

[14] Massoudi, M., Maneschy, C.E.: Numerical solution to the flow of a second grade fluid over a stretching sheet using the method of quasilinearization. Appl. Math. Comput. 149, 175-186, (2004)

[15] V. Kumaran, G. Ramanaiah, A note on the flow over a stretching sheet. Acta Mech. 116 (1996) 229-233.

[16] F.M. Ali, R. Nazar, N.M. Arifin, A. Ishak, I. Pop, Unsteady flow across a stretching surface. Int. Commun. Heat Mass 37 (2010) 476-479. 